\title{
Spin alignments of vector mesons in deeply inelastic lepton-nucleon scattering
}

\author{
Xu Qing-hua and Liang Zuo-tang \\ Department of Physics, Shandong University, Ji'nan, Shandong 250100, China
}

\begin{abstract}
We extend the calculations of the spin alignments of vector mesons in $e^{+} e^{-}$annihilation in a recent Rapid Communication to deeply inelastic lepton-nucleon scatterings. We present the results for different mesons in the current fragmentation regions of $\mu^{-} N \rightarrow \mu^{-} V X$ at high energies and $\nu_{\mu} N \rightarrow \mu^{-} V X$ at both high and low energies. We also present the predictions for $\nu_{\mu} N \rightarrow \mu^{-} V X$ at NOMAD energies in the target fragmentation region using a valence quark model.
\end{abstract}

PACS: 12.15.Ji, 13.60.Le, 13.87.Fh, 13.88.+e

Spin alignments of vector mesons in high energy reactions have attracted much attention recently $[1-8]$. Since the influences from decay of heavier hadrons are relatively small and the production rate is in general higher than that of hyperon, such studies provide important information for the spin effects in the fragmentation process, in particular the spin transfer from the fragmenting quark to the produced hadrons. Measurements have been carried out in different reactions [1- 1 in particular in $e^{+} e^{-}$annihilation at LEP recently [- The data show that the vector mesons produced in $e^{+} e^{-}$annihilation at $Z^{0}$ pole have a large probability to be in the helicity zero state, and the effect is more significant for large momentum fraction region.

In a recent paper [8], we calculated the spin density matrix of vector mesons in $e^{+} e^{-}$annihilation at $Z^{0}$ pole by taking the spin of vector meson which contains the fragmenting quark as the sum of the spin of the polarized fragmenting quark (antiquark) and that of the antiquark (quark) created in the fragmentation process. Compared with the data [ $[$, we showed that the experimental results for $\rho_{00}$, i.e., the probability for vector meson in the helicity zero state, imply a significant polarization for the antiquark (quark) which is created in the fragmentation process and combines with the fragmenting quark to form the vector meson. It should be polarized in the opposite direction as that of the fragmenting quark and the polarization can approximately be written as,

$$
P_{z}=-\alpha P_{f},
$$

where $\alpha \approx 0.5$ is a constant for most of the vector mesons. (Here, $P_{z}$ is the polarization of the antiquark in the moving direction of the fragmenting quark and $P_{f}$ is the longitudinal polarization of the fragmenting quark of flavor f) Using this result, we were able to fit the data of $\rho_{00}$ 's for different vector mesons reasonably. The relation given by Eq. (1) can be considered as a direct implication of

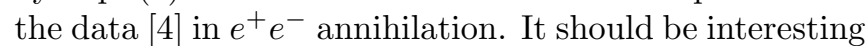
to extend the studies to other reactions in particular to check whether the relation shown in Eq. (1) is also true for the fragmentation of quarks in other processes. In this connection, it is encouraging to see that not only some previous data are available but also new measurements [9] can be made by NOMAD collaboration in $\nu_{\mu} N \rightarrow \mu^{-} H X$.
In this paper, we extend the calculations to deeply inelastic lepton-nucleon scatterings (DIS) and present the results for $\rho_{00}$ 's of different mesons in different cases. We now start our calculations by summarizing the main points of the method in Ref. [8]. To calculate the density matrix of vector mesons which are produced in the fragmentation of a polarized quark $q_{f}^{0}$, we divide them into the following two groups and consider them separately: (a) those which contain the fragmenting quark $q_{f}^{0}$; (b) those which don't contain the fragmenting quark. The spin density matrix $\rho^{V}\left(x_{F}\right)$ for the vector meson $V$ is given by:

$$
\rho^{V}\left(x_{F}\right)=\sum_{f} \frac{\left\langle n\left(x_{F} \mid a, f\right)\right\rangle}{\left\langle n\left(x_{F}\right)\right\rangle} \rho^{V}(a, f)+\frac{\left\langle n\left(x_{F} \mid b\right)\right\rangle}{\left\langle n\left(x_{F}\right)\right\rangle} \rho^{V}(b),
$$

where $\left\langle n\left(x_{F} \mid a, f\right)\right\rangle$ and $\rho^{V}(a, f)$ are the average number and spin density matrix of vector mesons from (a); $\left\langle n\left(x_{F} \mid b\right)\right\rangle$ and $\rho^{V}(b)$ are those from $(\mathrm{b}) ;\left\langle n\left(x_{F}\right)\right\rangle=$ $\sum_{f}\left\langle n\left(x_{F} \mid a, f\right)\right\rangle+\left\langle n\left(x_{F} \mid b\right)\right\rangle$ is the total number of vector mesons and $x_{F}$ is defined as $x_{F} \equiv 2 p_{z}^{*} / W, p_{z}^{*}$ is the momentum of vector meson in the $z$ direction in the centerof-mass system of total hadronic system, $W$ is the total energy of the hadronic system, and the $z$-axis is taken as the direction of the intermediate boson. The average numbers $\left\langle n\left(x_{F} \mid a, f\right)\right\rangle$ and $\left\langle n\left(x_{F} \mid b\right)\right\rangle$ are determined by the hadronization mechnism and can be calculated using hadronization models 10 as implemented by the MonteCarlo event generators.

The vector mesons from group (b) are taken as unpolarized, thus $\rho^{V}(b)=1 / 3$. For those from group (a) which contain $q_{f}^{0}$ and an antiquark $\bar{q}$ created in the fragmentation [11], the spin density matrix $\rho^{V}(a, f)$ is calculated from the direct product of the spin density matrix $\rho^{q_{f}^{0}}$ for $q_{f}^{0}$ and $\rho^{\bar{q}}$ for $\bar{q}$. Transforming the direct product, $\rho^{q_{f}^{0} \bar{q}}=\rho^{q_{f}^{0}} \otimes \rho^{\bar{q}}$, to the coupled basis $\left|s, s_{z}\right\rangle$ (where $\left.\vec{s}=\vec{s}^{q}+\vec{s}^{\bar{q}}\right)$, we obtain the spin density matrix $\rho^{V}(a, f)$, and the 00-component as,

$$
\rho_{00}^{V}(a, f)=\left(1-P_{f} P_{z}\right) /\left(3+P_{f} P_{z}\right),
$$

where $P_{f}$ is the longitudinal polarization of $q_{f}^{0}$ and $P_{z}$ is the polarization of $\bar{q}$ in $z$-direction. We insert the relation showed by Eq. (11) into Eq. (3) and obtain 


$$
\rho_{00}^{V}(a, f)=\left(1+\alpha P_{f}^{2}\right) /\left(3-\alpha P_{f}^{2}\right) .
$$

Finally, from Eqs. (2) and (4), we have

$$
\rho_{00}^{V}\left(x_{F}\right)=\sum_{f} \frac{1+\alpha P_{f}^{2}}{3-\alpha P_{f}^{2}} \frac{\left\langle n\left(x_{F} \mid a, f\right)\right\rangle}{\left\langle n\left(x_{F}\right)\right\rangle}+\frac{1}{3} \frac{\left\langle n\left(x_{F} \mid b\right)\right\rangle}{\left\langle n\left(x_{F}\right)\right\rangle} .
$$

Using this result, we obtained [8] a good fit to the data of $\rho_{00}^{V}$ 's in $e^{+} e^{-}$annihilation. Now, we apply it to the DIS processes.

We first consider the process of $\mu^{-} p \rightarrow \mu^{-} V X$. For a sufficiently large $W$, the hadrons produced in the current fragmentation region are mainly from the struck quark's fragmentation. Considering only the leading order subprocess $\mu^{-} q \rightarrow \mu^{-} q$, the polarization of the outgoing struck quark can be obtained from QED and can be found in different publications [see,e.g. 12], i.e.,

$$
P_{f}=\frac{P^{l} D_{L}(y) q_{f}(x)+P^{N} \Delta q_{f}(x)}{q_{f}(x)+P^{l} D_{L}(y) P^{N} \Delta q_{f}(x)},
$$

where $D_{L}(y)$ is the longitudinal depolarization factor $D_{L}(y)=\left[1-(1-y)^{2}\right] /\left[1+(1-y)^{2}\right]$ and $y \equiv p \cdot\left(k-k^{\prime}\right) / p \cdot k$ where $p, k$, and $k^{\prime}$ are the four momenta of the incoming $q, \mu^{-}$, and the outgoing $\mu^{-}$respectively. $P^{l}$ and $P^{N}$ are the longitudinal polarizations of the incoming lepton and nucleon respectively, $q_{f}(x)$ and $\Delta q_{f}(x)$ are the unpolarized and longitudinally polarized distribution functions. In our calculation, the unpolarized and longitudinal polarized distribution functions are taken as the GRV98 LO set 13] and the standard LO scenario of GRSV2000 14] respectively.

We use the generator LEPTO [15] to calculate the average numbers $\left\langle n\left(x_{F} \mid a, f\right)\right\rangle$ and $\left\langle n\left(x_{F} \mid b\right)\right\rangle$. As an example, we show the different orgions of $K^{*+}$ in the current fragmentation region of $\mu^{-} N \rightarrow \mu^{-} K^{*+} X$ at the beam energy of $500 \mathrm{GeV}$ in Fig. 1. We choose the events for $Q^{2}>5$ $(\mathrm{GeV} / \mathrm{c})^{2}, 10^{-4}<x<0.2$, and $0.5<y<0.9$ to ensure a reasonably large $W$ and a reasonably high polarization of the fragmenting quark. We see that, in contrast to hyperon production in the same reaction [12], the decay contribution is indeed very small.

Using Eq.(5) and the average numbers obtained above, we calculate the $\rho_{00}^{V}$ 's for $K^{* 0}, K^{*+}, \rho^{ \pm}$and $\rho^{0}$. The results are shown in Fig. 2 for different combinations of $P^{l}$ and $P^{N}$ in the same kinematic region as in Fig. 1. We see that $\rho_{00}^{V}$ 's increase with increasing $x_{F}$ and reach about 0.5 in the cases of polarized lepton beam, which is much larger than $1 / 3$ and can easily be detected in experiments. However, the magnitude of $\rho_{00}^{V}$ 's for $P^{l}=0$ and $P^{N}=1$ is much smaller than those in other three cases. The reason is that the ratio $\Delta q_{f}(x) / q_{f}(x)$ is small in the chosen small $x$ region, so $P_{f}$ obtained from Eq.(6) is small. While the large $D_{L}(y)$ for large $y$ region leads to relative large $P_{f}$ in other three cases, the result of which is large $\rho_{00}^{V}$. Hence, to get reasonably large $\rho_{00}^{V}$, the polarization of the lepton beam is required.
For inclusive meson production in unpolarized $e p$ reactions, the struck quark is unpolarized. Hence, $P_{f}=0$ and $\rho_{00}^{V}=1 / 3$ in the current fragmentation region. There have been measurements [1] for $\rho^{0}$ with $z>0.4\left(z=E_{\rho} / \nu\right.$ and $\nu$ is the energy loss of the lepton beam in the lab frame) in inelastic $e p$ scattering at $E_{e}=11.5 \mathrm{GeV}$ and the result is $\rho_{00}=0.41 \pm 0.08$, which is in agreement with the theoretical expectation.

For $\nu_{\mu} N \rightarrow \mu^{-} V X$, the leading subprocess $\nu_{\mu} q \rightarrow \mu^{-} q$ is a charged current weak interaction with the exchange of a virtual $W^{+}$, which selects only left-handed quarks or right-handed antiquarks. Thus, $P_{f}=-1$ for struck quarks and $P_{f}=1$ for struck antiquarks, whose polarizations reach the maxmum. Hence, we expect larger $\rho_{00}^{V}$ 's in neutrino DIS than in other reactions. We calculate $\rho_{00}^{V}$ 's for different mesons in the current fragmentation region of $\nu_{\mu} N \rightarrow \mu^{-} V X$ at $E_{\nu}=500 \mathrm{GeV}$ and the results are shown in Fig. 3. We see that, the $\rho_{00}^{V}$ 's for $K^{*+}, \rho^{+}$ and $\rho^{0}$ increase to about 0.6 with increasing $x_{F}$. However, the $\rho_{00}$ for $K^{* 0}$ is much smaller. This is because, for $K^{* 0}$, the only contribution of type (a) is from outgoing struck $\bar{s}$ quark which is a result of the absorption of $W^{+}$by a $\bar{c}$ in the nucleon sea. It is very small due to the rarity of $\bar{c}$ in the nucleon. Other kinds of contributions lead only to $1 / 3$ for $\rho_{00}$. For other vector mesons, such as $K^{*-}$ and $\rho^{-}$, there is no contribution of type (a) at all. Their $\rho_{00}$ 's are just equal to $1 / 3$.

It has been pointed out that [16], at lower energies, such as at the NOMAD energies where $\left\langle E_{\nu}\right\rangle=43.8 \mathrm{GeV}$, the influence of the fragmentation of target remnant to hyperon production is very large, in particular in the small $x_{F}$ region [16]. It has to be taken into account in calculating the hyperon polarization in such energy region. The characteristic features of the hyperon polarization in this region are determined by this contribution. It is therefore natural to ask whether similar effects also exist for mesons production. To check this, we make an analysis using LEPTO and the results for $\nu_{\mu} p \rightarrow \mu^{-} K^{*+} X$ at $E_{\nu}=43.8 \mathrm{GeV}$ are shown in Fig. 4 . We see that, there is indeed a mixture of the contribution from the mesons containing the struck quark and that containing one of the quarks in the target remnant in the region near $x_{F} \approx 0$, but the effect is much smaller than that for hyperon production. This can be understood easily. We recall that, in the case of hyperon production, the excitation of diquark-anti-diquark pair is needed for producing hyperons which contain the struck quark and there should be at least one more baryon and one antibaryon produced. However, the excitation of diquark-anti-diquark pair is unnecessary for producing hyperons which contain one quark of the remnant uu diquark. At the NOMAD energies, $W$ is only of several $\mathrm{GeV}$, the probability for the former case should be much smaller than that for the latter. Hence, in the $x_{F} \sim 0$ region, the contributions from the latter case can dominate. For meson production, to produce mesons containing the struck quark or one $u$ 
quark of the remnant uu diquark, only quark-antiquark pair excitation is needed. The probability for the mesons containing one quark of $u u$ diquark to move in the opposite direction of the diquark is rather small. Hence, the influences from target remnant fragmentation on the spin alignments of vector mesons in current fragmentation region are small.

Having the above-mentioned results for meson production of different origins, we can also calculate their spin alignments by taking the fragmentation of the nucleon remnant into account. The polarization of the quarks in target remnant is unclear. We calculate it in the same way as in Ref. 16] in studying hyperon polarization, where a valence quark model was used. The results obtained for $\rho_{00}^{V}$ 's in $\nu_{\mu} p \rightarrow \mu^{-} V X$ at $E_{\nu}=43.8 \mathrm{GeV}$ both in the current and target fragmentation regions are shown in Fig. 5. We see that, for $x_{F}>0$, the influence from the target remnant fragmentation is indeed very small. Compared with those obtained at $E_{\nu}=500 \mathrm{GeV}, \rho_{00}$ of $K^{* 0}$ is smaller. This is because at such low energy, the probability for the outgoing struck quark to be $\bar{s}$ is very tiny. For the target fragmentation region, the spin alignments are smaller than those in the current region. The results for a neutron target are shown in Fig. 6. The $\rho_{00}^{V}$ 's are much smaller in the target fragmentation region than those in case of a proton target, because the polarization of the quark in the remnant $u u$ diquark from a proton is larger than that in the remnant $u d$ diquark from a neutron [16]. The difference in the current region is tiny for the two different targets, since the partonic subprocesses are the same and $\nu_{\mu} d \rightarrow \mu^{-} u$ dominates others for both targets.

There have been measurements [2] for $\rho^{0}$ in neutrino DIS and the results are $\rho_{00}=0.65 \pm 0.18$ and $0.61 \pm 0.08$ in $\bar{\nu} N_{e} \rightarrow \mu^{+} \rho^{0} X$ and $\rho_{00}=0.41 \pm 0.13$ and $0.39 \pm 0.08$ in $\nu N_{e} \rightarrow \mu^{-} \rho^{0} X$ at low energies similar to NOMAD. The data are both for $\rho^{0}$ 's with $z>0.4\left(z=E_{\rho} / \nu\right)$. Our results in the same kinematic region are $\rho_{00}=0.511$ for $\bar{\nu} N_{e} \rightarrow \mu^{+} \rho^{0} X$ and $\rho_{00}=0.518$ for $\nu N_{e} \rightarrow \mu^{-} \rho^{0} X$ [17. They are in agreement with the data and further measurements with high precision are required to give a better check of the model.

In summary, we calculate the spin alignments for different vector mesons in the current fragmentation regions of $\mu^{-} N \rightarrow \mu^{-} V X$ and $\nu_{\mu} N \rightarrow \mu^{-} V X$ at high energies by taking the spin of a vector meson as the sum of the spins of the polarized fragmenting quark (antiquark) and that of the antiquark (quark) created in the fragmentation process. We also present the predictions for spin alignments in $\nu_{\mu} N \rightarrow \mu^{-} V X$ at NOMAD energies both in the current and target fragmentation regions. The results show that there are significant spin alignments for most of the vector mesons in the above reactions. Measurements of them can provide important information for the spin effects in the fragmentation process.

We thank Li Shi-yuan, Liu Chun-xiu, Xie Qu-bing and other members in the theoretical particle physics group of
Shandong University for helpful discussions. This work was supported in part by the National Science Foundation of China (NSFC) and the Education Ministry of China under Huo Ying-dong Foundation.

[1] I. Cohen. et al., Phys. Rev. D 25, 634, (1982).

[2] BEBC WA59 Collab., W. Witttek et al., Phys. Lett. B187, 179 (1987); BBCN Collab., V.G. Zaetz et al., Z. Phys. C66, 583 (1995).

[3] EXCHARM Collab., A. N. Aleev et al., Phys. Lett. B485, 334 (2000).

[4] DELPHI Collab., P. Abreu et al., Phys. Lett. B406, 271 (1997); OPAL Collab., K. Ackerstaff et al., Phys. Lett. B412, 210 (1997); OPAL Collab., K. Ackerstaff et al., Z. Phys. C74, 437 (1997); OPAL Collab., G. Abbiendi et al., Eur. Phys. J. C16, 61 (2000).

[5] A.F. Falk and M.E. Peskin, Phys. Rev. D 49, 3320(1994).

[6] M. Anselmino, M. Bertini, F. Murgia and B. Pire, Phys. Lett. B438, 347 (1998); M. Anselmino, M. Bertini, F. Murgia and P. Quintairos, Eur. Phys. J. C11, 529 (1999).

[7] J. P. Ma, Nucl. Phys. B622, 416 (2002).

[8] Xu Qing-hua, Liu Chun-xiu and Liang Zuo-tang, Phys. Rev. D 63, 111301(R) (2001).

[9] D. Naumov, private communication (2001).

[10] B. Andersson, G. Gustafson, G. Ingelman, and T. Sjöstrand, Phys. Rep. 97, 31 (1983).

[11] It is similar for those of type (a) from the fragmentation of a polarized antiquark $\bar{q}_{f}^{0}$, i.e., $V=\left(\bar{q}_{f}^{0} q\right)$.

[12] Liu Chun-xiu, Xu Qing-Hua, and Liang Zuo-tang, Phys. Rev. D 64, 073004 (2001).

[13] M. Glück, E. Reya, and A. Vogt, Eur. Phys. J. C 5, 461 (1998).

[14] M. Glück, E. Reya, M. Stratmann, and W. Vogelsang, Phys. Rev. D 63, 094005 (2001).

[15] G. Ingelman, A.Edin, J.Rathsman, Comp. Phys. Comm. 101, 108 (1997).

[16] Liang Zuo-tang and Liu Chun-xiu, hep-ph/0204323, submitted to Phys. Rev. D; See also, Liang Zuo-tang, talks at 31st Int. Sym. on Mitiparticle-dynamics (ISMD31) and 3rd Circum-Pan-Pacific Symposium on High Energy Spin Physics, hep-ph/0111403 and hep-ph/0205017.

[17] We note that the quantization axis of the data is chosen along the moving direction of the vector meson (helicity frame), while in our calculations it is chosen as the moving direction of the outgoing struck quark. There is a slight difference between the results in these two frames [8] and the magnitudes of the differences depend on the transverse momentum of the meson with respect to the outgoing struck quark and other related factors. We estimate that, typically, the correction is within 0.05 . 


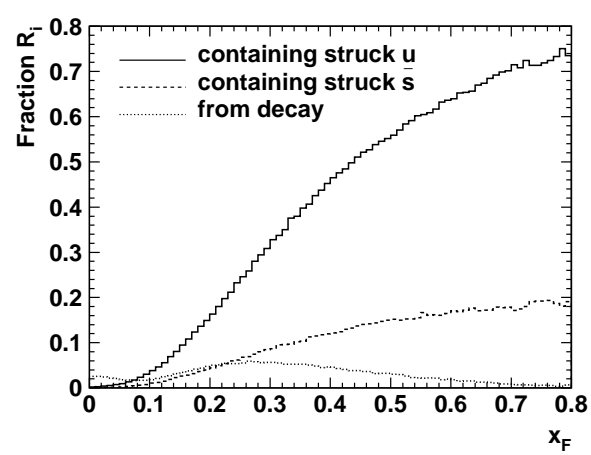

FIG. 1. Different contributions to $K^{*+}$ production in the current region of $\mu^{-} N \rightarrow \mu^{-} K^{*+} X$ at $E_{\mu}=500 \mathrm{GeV}$.

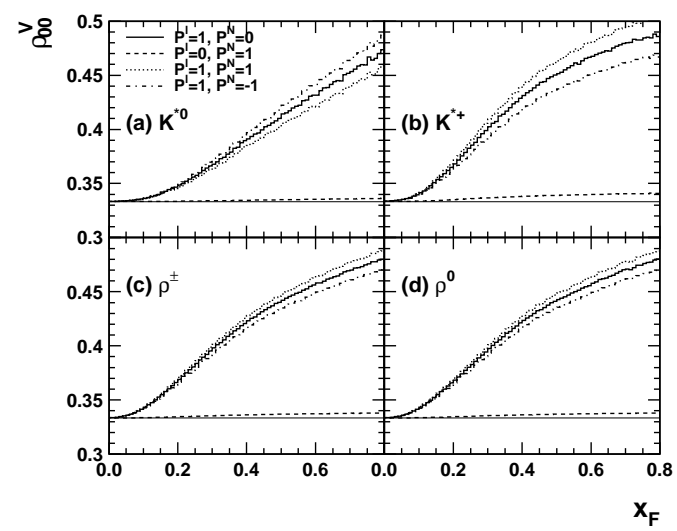

FIG. 2. $\rho_{00}^{V}$ in the current region of $\mu^{-} p \rightarrow \mu^{-} V X$ at $E_{\mu}=500 \mathrm{GeV}$. The straight lines at $\rho_{00}=1 / 3$ show the unpolarized cases.

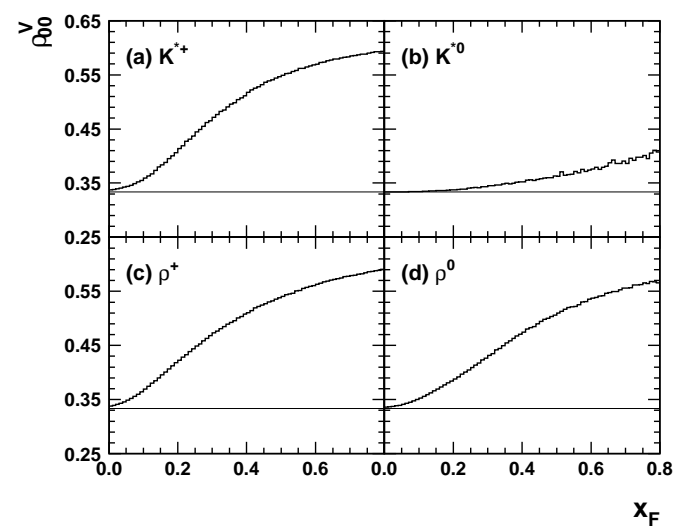

FIG. 3. $\rho_{00}^{V}$ in the current region of $\nu_{\mu} p \rightarrow \mu^{-} V X$ at $E_{\mu}=500 \mathrm{GeV}$. The straight lines at $\rho_{00}=1 / 3$ show the unpolarized cases.

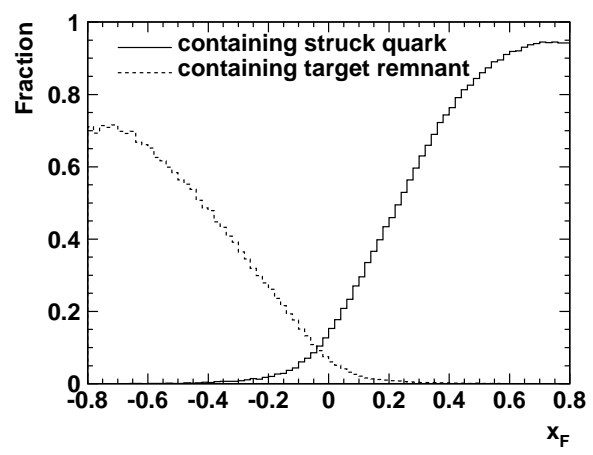

FIG. 4. Different contributions to $K^{*+}$ production both in the current and target fragmentation region of $\nu_{\mu} p \rightarrow \mu^{-} K^{*+} X$ at $E_{\nu}=43.8 \mathrm{GeV}$.

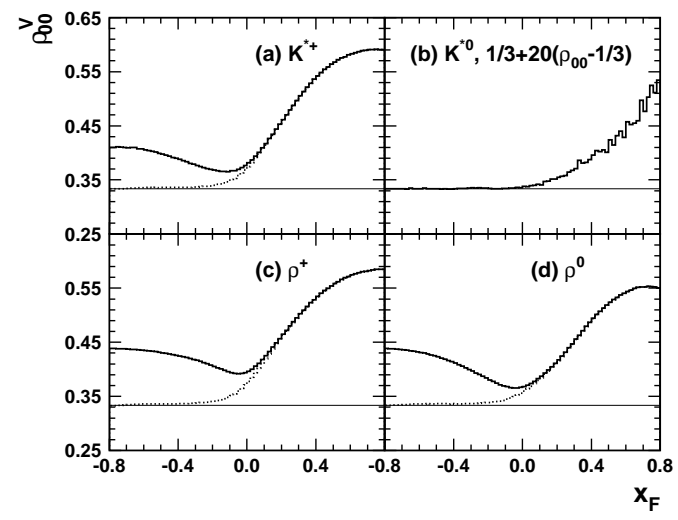

FIG. 5. $\rho_{00}^{V}$ in $\nu_{\mu} p \rightarrow \mu^{-} V X$ at $E_{\nu}=43.8 \mathrm{GeV}$. The solid line represents the results where the contribution of target fragmentation is taken into account, the dotted line denotes the results where only the contribution of the current fragmentation is included. The results for $K^{* 0}$ are same.

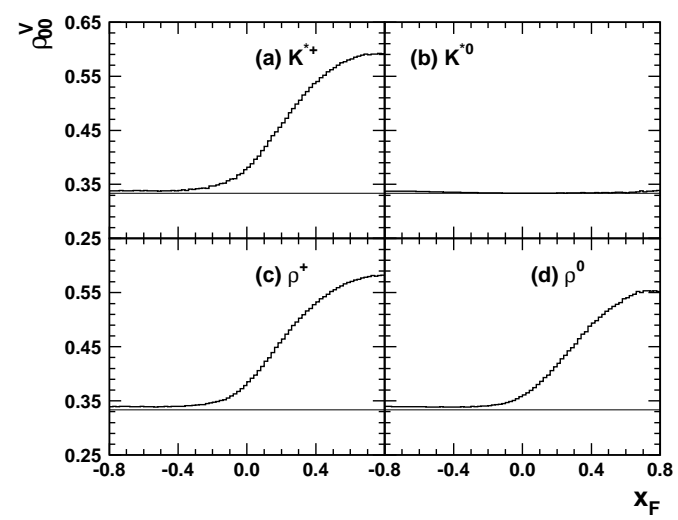

FIG. 6. $\rho_{00}^{V}$ in $\nu_{\mu} n \rightarrow \mu^{-} V X$ at $E_{\nu}=43.8 \mathrm{GeV}$. 